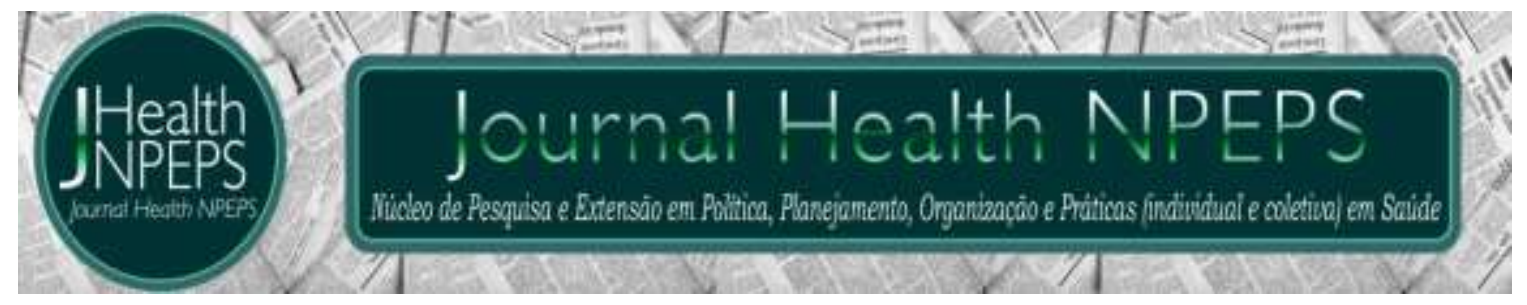

http://dx.doi.org/10.30681/252610104119

ARTIGO ORIGINAL

\title{
Sentimentos e expectativas de mulheres frente ao diagnóstico de câncer de mama
}

\section{Feelings and expectations of women regarding the diagnosis of breast cancer}

\section{Sentimientos y expectativas de las mujeres en el diagnóstico de cáncer de mama}

\author{
Ruth Silva Lima da Costa ${ }^{1}$, Reimilas dos Santos Maciel Lima ${ }^{2}$, Tamirez \\ Cordeiro Félix ${ }^{3}$, Thaís Milani Silva Cardoso da Mota ${ }^{4}$, Emily Assis \\ Tavares $^{5}$, Gleicy Janáira da Costa Queiroz ${ }^{6}$, Elizângela Pessoa Pereira ${ }^{7}$
}

\section{RESUMO}

Objetivo: evidenciar os sentimentos e expectativas de mulheres frente ao diagnóstico de câncer de mama. Método: trata-se de estudo descritivo com abordagem qualitativa, desenvolvido junto a 12 mulheres, diagnosticadas com câncer de mama, no ano de 2017 em Rio Branco - Acre, Brasil. Resultados: a maioria das mulheres pertenciam a faixa etária de 50 a 59 anos, com ensino médio completo, casadas e com renda familiar de 1 a 2 salários mínimos. Para grande parte delas, o primeiro sintoma da doença foi o aparecimento de nódulo mamário, com difícil aceite, pois revelaram medo da morte. As expectativas frente ao futuro após o diagnóstico eram de esperança e de que

\footnotetext{
${ }^{1}$ Enfermeira. Mestre em Ciências da Saúde. Enfermeira Auditora na Secretaria de Estado de Saúde do Acre e Docente nos cursos de Medicina e Enfermagem do Centro Universitário Uninorte. Rio Branco, Acre, Brasil. Email: ruttylyma@gmail.com ORCID ID: https://orcid.org/ 0000-0003-1890-086X Autor principal - Endereço para correspondência: Alameda Alemanha, 200 - Jardim Europa, Rio Branco - AC, 69915-901.

${ }^{2}$ Enfermeira. Graduada em Enfermagem pelo Centro Universitário Uninorte. Rio Branco, Acre, Brasil Email: reimilas@gmail.com ORCID ID: https://orcid.org/ 0000-0002-3823-4035

${ }^{3}$ Enfermeira. Graduada em Enfermagem pelo Centro Universitário Uninorte. Rio Branco, Acre, Brasil Email: tamirezfelix@gmail.com ORCID ID: https://orcid.org/ 0000-0001-5723-5503

${ }^{4}$ Enfermeira. Graduada em Enfermagem pelo Centro Universitário Uninorte. Rio Branco, Acre, Brasil Email: milaniscm@gmail.com ORCID ID: https://orcid.org/0000-0001-7808-6375

${ }^{5}$ Graduada em Enfermagem pelo Centro Universitário Uninorte. Rio Branco, Acre, Brasil E-mail: emily.assistavares@hotmail.com ORCID ID: https://orcid.org/ 0000-0001-7659-6793

${ }^{6}$ Graduada em Enfermagem pelo Centro Universitário Uninorte. Rio Branco, Acre, Brasil E-mail: gleicynaira123@gmail.com ORCID ID: https://orcid.org/ 0000-0002-9202-7205

${ }^{7}$ Graduada em Enfermagem pelo Centro Universitário Uninorte. Rio Branco, Acre, Brasil E-mail: elly.pessoa@gmail.com ORCID ID: https://orcid.org/0000-0001-6019-8862
} 
a cura iria chegar, além de sentimentos de gratidão por estar vencendo a doença e o desejo de compartilhar com outras mulheres sua experiência; fortalecê-las diante dos desafios no enfrentamento da doença. Conclusão: após a confirmação do diagnóstico, faz-se necessário a efetivação de ações multiprofissionais voltadas a mulher, especialmente em relação aos aspectos emocionais, para que ela possa vivenciar esse processo, sentindo-se mais forte e acolhida.

Descritores: Neoplasias da Mama; Emoções; Saúde da Mulher; Diagnóstico.

\section{ABSTRACT}

Objective: to evidence the feelings and expectations of women regarding the diagnosis of breast cancer. Method: this is a descriptive study with a qualitative approach, developed with 12 women diagnosed with breast cancer in 2017 in Rio Branco - Acre, Brazil. Results: most of the women belonged to the age group of 50 to 59 years, with complete high school, married and with family income of 1 to 2 minimum wages. For most of them, the first symptom of the disease was the appearance of a breast lump, which is difficult to accept, as they revealed fear of death. The expectations for the future after the diagnosis were of hope and that a cure would come, in addition to feelings of gratitude for overcoming the disease and the desire to share their experience with other women; strengthening them to the challenges facing the illness. Conclusion: after confirming the diagnosis, it is necessary to carry out multiprofessional actions aimed at women, especially in relation to emotional aspects, so that they can experience this process feeling more welcomed and stronger.

Descriptors: Breast Neoplasms; Emotions; Women's Health; Diagnosis.

\section{RESUMEN}

Objetivo: evidenciar los sentimientos y expectativas de las mujeres con respecto al diagnóstico de cáncer de seno. Método: este es un estudio descriptivo con un enfoque cualitativo, desarrollado con 12 mujeres, diagnosticadas con cáncer de seno, en 2017 en Rio Branco - Acre, Brasil. Resultados: la mayoría de las mujeres pertenecían al grupo de edad de 50 a 59 años, con educación secundaria completa, casadas y con ingresos familiares de 1 a 2 salarios mínimos. Para la mayoría de ellos, el primer síntoma de la enfermedad fue la aparición de un bulto en el seno, que es difícil de aceptar, ya que revelaron temor a la muerte. Las expectativas para el futuro después del diagnóstico eran esperanzadoras y que vendría una cura, además de los sentimientos de gratitud por haber superado la enfermedad y el deseo de compartir su experiencia con otras mujeres; fortalecerlos frente a los desafíos para hacer frente a la enfermedad. Conclusión: después de confirmar el diagnóstico, es necesario llevar a cabo acciones multiprofesionales dirigidas a las mujeres, especialmente en relación con los aspectos emocionales, para que pueda experimentar este proceso sintiéndose más fuerte y bienvenida.

Descriptores: Neoplasias de mama; Emociones; Salud de la mujer;

Diagnóstico.

\section{INTRODUÇÃO}


Sentimentos e expectativas de mulheres...

O câncer de mama é o segundo tipo mais frequente entre as mulheres do mundo. Com estimativa de 59.700 casos novos e risco estimado de 56,20 casos a cada 100 mil mulheres por ano ${ }^{1}$. Ele é mais prevalente no sexo feminino, acometendo prioritariamente mulheres com idade entre 40 e 69 anos. No Brasil, é o tipo mais comum de câncer, representando a principal causa de mortalidade entre mulheres ${ }^{2,3}$. No Estado do Acre, até então, não existem estudos recentes que possam evidenciar a epidemiologia da doença.

\section{Algumas}

condições

referentes ao estilo de vida tendem a aumentar os fatores de risco para - câncer de mama, tais como, predisposição genética, realização de intervenção hormonal, maternidade após os 30 anos, sedentarismo, má alimentação, obesidade e uso/abuso de álcool e outras drogas. Além disso, mulheres com idade a partir dos 50 anos, apresentam maior risco de desenvolver a doença ${ }^{4}$.

As mulheres que apresentam um elevado risco para o desenvolvimento do câncer de mama, devem receber atenção especial por parte da equipe de saúde, para a incorporação de estratégias de prevenção, diagnóstico e tratamento precoce, visando melhores prognósticos. Para isso, a equipe deve estar sensibilizada e preparada para proporcionar esse acompanhamento integral ${ }^{5}$.

Todavia, diante do diagnóstico da doença, inevitavelmente a mulher sofre grande impacto psicoemocional, com vivência de sentimentos de medo, tristeza, dor, angústia, revolta, insegurança e incertezas frente a essa nova realidade. Esse momento requer apoio dos profissionais e familiares para tais enfrentamentos ${ }^{6,7}$.

Apesar de reconhecer essas nuances emocionais, alguns tipos de câncer podem desencadear emoções distintas, seja pelo tempo de evolução da doença, acesso ao tratamento, como pela familiaridade e aceitação social. Assim, este estudo objetivou evidenciar os sentimentos e expectativas de mulheres após serem diagnosticadas com câncer de mama. 
MÉTODO

Trata-se de estudo descritivo com abordagem qualitativa, desenvolvido junto a 12 mulheres, diagnosticadas com câncer de mama, no ano de 2017. A coleta foi realizada em outubro de 2018, a partir de registros de mulheres assistidas na unidade de assistência de alta complexidade (UNACON) de um hospital de médio porte da cidade de Rio Branco (AC), Brasil. Optou-se por esta instituição, por ser a única do Estado que realiza tratamento oncológico.

Os critérios de inclusão foram: mulheres diagnosticadas com câncer de mama no ano de 2017. Foram excluídas aquelas que possuíam histórico anterior de qualquer tipo de câncer.

Para a coleta de dados, foi elaborado um questionário contendo 12 questões abertas e fechadas que visavam avaliar o perfil sociodemográfico das mulheres, bem como os sentimentos e expectativas frente ao diagnóstico do câncer de mama.
A amostra foi composta por 12 mulheres que foram selecionadas pelo método de seleção aleatória simples e saturação. Os dados de identificação foram coletados através de informações inseridas em seus prontuários. Após essa fase, os pesquisadores entraram em contato prévio por via telefônica, marcando melhor data e horário para a realização da coleta de dados. As entrevistas ocorreram no domicílio das mulheres e tiveram duração média de 40 minutos.

A conversação foi gravada e, posteriormente, transcrita na íntegra em arquivo eletrônico. 0 tratamento dos dados foi realizado através da Análise de Conteúdo de Bardin $^{8}$, compondo-se de leituras flutuantes, que levou ao agrupamento de informações e categorização dos dados. Mediante a análise dos depoimentos das participantes do estudo, os achados foram agrupados em três categorias: "Aparecimento dos primeiros sintomas da doença", "Sentimentos frente a descoberta do câncer" e "Expectativas sobre o futuro após o diagnóstico de câncer de mama". 
Com o intuito de preservar a identidade das participantes, de acordo com a Resolução 466/12 que trata da pesquisa com seres humanos, foi utilizada o nome de pássaros nas citações das falas, a saber: Calopsita, Beija Flor, Bem Te Vi, Garça, Pardal, Sabiá, Andorinha, Tico Tico, Gaivota, Arara e Águia.

A pesquisa foi aprovada pelo Comitê de Ética e pesquisa do Centro Universitário União Educacional do Norte sob o parecer de $\mathrm{n}^{\circ}$ 3.096.167 como também pelo Comitê de Ética e Pesquisa do Hospital das Clínicas do Acre HCA/FUNDHACRE sob parecer de aprovação $\mathrm{n}^{\circ} 3.235 .844$ e CAAE: 99389218.4.3001.5009.

\section{RESULTADOS E DISCUSSÃO}

A maioria das mulheres desse estudo encontrava-se na faixa etária de 50 a 59 anos, casadas, com ensino médio completo e renda familiar entre 1.045,00 a 2.000,00 reais. Resultados semelhantes foram encontrados em estudo sobre o perfil sócio demográfico de mulheres diagnosticadas com câncer de mama, realizado em São Paulo (SP), onde a faixa etária mais predominante foi entre 50 e 69 anos $(44,15 \%)$, casadas $(26,4 \%)$ e com escolaridade até 0 ensino fundamental completo $(44,7 \%)^{9}$. Resultado divergente referente a idade, foi evidenciado em um estudo realizado na Eslováquia (SK) onde observou-se aumento significativo de casos de câncer de mama na população jovem e entre mulheres na pré-menopausa ${ }^{10}$.

Outro estudo realizado com 80 mulheres

também diagnosticadas com câncer de mama em Fortaleza (CE), demostrou que $68 \%$ estavam na faixa etária de 40 a 49 anos, 59\% eram casadas, $68 \%$ também possuíam o ensino médio completo e a renda familiar predominante era de 1 e 2 salários mínimos ${ }^{11}$, em consonância com os resultados encontrados no presente estudo.

Uma pesquisa desenvolvida pela American Câncer Society em 2015, nos Estados Unidos, concluiu que a probabilidade de se desenvolver câncer de mama aumenta com o passar dos anos, sendo $1,9 \%$ até 49 anos, $2,3 \%$ de 50 59 anos, 3,5\% de 60 a 69 anos e 
6,7\% para mulheres com idades acima de 70 anos. Essa mesma pesquisa confirmou que 0 sedentarismo, tabagismo e a ingestão de bebidas alcoólicas, impactam diretamente na ocorrência da doença, em mais de 90\% dos casos, além do histórico familiar ${ }^{12}$.

\section{Aparecimento dos primeiros} sintomas da doença

Para a maioria das mulheres investigadas nesse estudo, o primeiro sintoma do câncer foi identificado através de um nódulo na mama, após a realização do autoexame.

Bom quando começou, começou um caroço, e o caroço foi crescendo, crescendo e foi crescendo né, quando eles vieram me atender o caroço já tava do tamanho de uma laranja. (Pardal, 49 anos)

Como já tinha casos na família sempre me preocupei, até que um dia sentia muito dor na mama e descobri o nódulo. (Beija Flor, 56 anos)

No começo eu senti umas pontadas bem fortes no seio, ai pensei meu Deus o que será isso, fui fazer uma palpação e não deu outra encontrei um caroço que já estava era grande $e$ eu nunca tinha percebido.

(Tico Tico, 54 anos)

De acordo com dados do INCA em 2019, o nódulo é o principal sintoma de câncer a ser notado pelas mulheres. Ele é indolor e fixo, e $90 \%$ das mulheres sempre o identificam primeiro em relação a outros sintomas ${ }^{13}$. Achados de um estudo semelhante sobre o aparecimento do câncer de mama em mulheres no estado de São Paulo (SP), demostrou que $78 \%$ delas também relataram sentir um nódulo palpável como queixa principal ${ }^{14}$.

É possível que a mulher identifique os primeiros sinais do câncer de mama por meio da observação do próprio corpo e do autoexame. Desta forma, a mesma pode ser impulsionada a buscar exames para a confirmação e possibilitar o diagnóstico precoce $^{15,16}$.

Frente aos sinais e sintomas da doença, é importante que a equipe de saúde esteja preparada e possa orientar as mulheres quanto aos seus sinais iniciais, bem como oferecer um cuidado integral que extrapole a condição do diagnóstico do câncer ${ }^{17}$. 
Um estudo realizado na Inglaterra frente a mulheres diagnosticadas com câncer de mama na atenção primária, identificou entre elas um total de 56 sintomas para um possível diagnóstico. Dentre esses, o nódulo mamário foi o mais frequente $(83 \%)$, seguido de sintomas não mamários, por exemplo, anormalidades mamilares (7\%) e dor mamária (6\%); e sintomas não relacionados à mama, como, dor nas costas (1\%) e perda de peso $(0,3 \%)$. 0 estudo evidenciou ainda que as mulheres que apresentam sintomas não mamários tendem a demorar na busca por ajuda, levando a um atraso no diagnóstico e tratamento ${ }^{18}$.

Atualmente, em todo o mundo, as campanhas de prevenção ao câncer têm primariamente focado no nódulo mamário como o primeiro sintoma da doença. No entanto, uma vez que os sintomas não mamários também são bastante representativos e que levam a um diagnóstico tardio e consequentemente menores chances de sucesso no tratamento, faz-se necessário que as campanhas educativas e os estudos científicos passam focar também na categoria de sintomas mamários "não nódulos", para que haja maior controle e melhor prognóstico da doença ${ }^{19-21}$.

\section{Sentimentos frente a descoberta do câncer}

Os discursos das mulheres revelaram o grande impacto frente ao recebimento do diagnóstico. A maioria afirmou não aceitar no primeiro momento, porém algumas verbalizaram que de alguma forma já esperavam pela confirmação, pelo fato de já possuírem casos na família. Outras ainda, mencionaram medo da morte e tristeza profunda, mas também a vontade de lutar pela cura.

Foi o momento mais difícil da minha vida que eu tive que passar, porque eu acho que nenhuma mulher gostaria de passar por isso. Aquele momento eu tive vontade de chorar muito, eu não aceitei, tive muito medo de morrer e deixar minha casa, meus filhos $e$ meus netinhos. (Águia, 50 anos)

Minha filha, descobrir uma doença como essa não é fácil não, eu senti muita dor no coração, muita tristeza, fiquei com medo de morrer, de não ter forças para lutar, medo de tudo. (Gaivota, 45 anos) 
Sentimentos e expectativas de mulheres...

No fundo eu já sabia que ia dar positivo, minha mãe teve e eu que cuidei dela, então eu já estava preparada para a notícia porque já sabia como é que era, difícil foi né, demais, porque eu sou muito nova com uma doença dessa, tenho meu esposo, meu trabalho e meus filhos, mas eu tive muita vontade de lutar, minha mãe conseguiu vencer $e$ porque eu não ia conseguir vencer também né. (Sabiá, 36 anos)

Ao receber o diagnóstico, a maioria das mulheres pode reagir recusando a descoberta, passando por vários estágios psicoemocionais $^{18}$. Esses achados estão de acordo com estudo realizado em Botucatu (SP) que evidenciou que ao receber 0 diagnóstico de câncer de mama, além do sentimento de negação, as mulheres demostram sinais de desespero e auto conflito 22 .

$$
\text { Assim como, }
$$

participantes que já esperavam pela confirmação do diagnóstico, estudo realizado na cidade de Londrina (PR), também encontrou 0 mesmo resultado, uma vez que as mulheres verbalizaram que devido a condição hereditária e pela percepção do próprio corpo, o diagnóstico positivo, de certa forma já era previsível ${ }^{23}$.

Frente as emoções desencadeadas após a confirmação do diagnóstico, o medo da morte é um sentimento comum, seguido da incerteza quanto ao futuro e a resposta ao tratamento ${ }^{24}$. Alguns estudos que também abordaram essa questão, identificaram ainda que a notícia da positividade da doença gera sentimento de impotência, ansiedade, inconformismo, tristeza, angústia, preocupação com o tratamento e receio da solidão. Essa somatória de sentimentos, leva a sensação de que a vida pode estar chegando ao fim, sendo imprescindível que elas recebam apoio emocional para enfrentar esse momento ${ }^{25-28}$.

Estudo com mulheres recém-diagnosticadas com câncer de mama na Inglaterra, identificou que 0 resultado positivo para a doença, trouxe uma resposta diferente para cada uma delas. Dentre essas, a maioria não havia sido preparada previamente para o resultado, demostraram excesso de preocupação com a doença e a necessidade não atendida de controle da fadiga. Para outras, o mais relevante foi o impacto da adaptação a sua nova condição, relatando extrema tristeza devido à perda dos cabelos, com queda da 
autoestima. Além disso, também observaram a evidente necessidade de aconselhamento sobre saúde sexual, reprodutiva e o relacionamento conjugal a partir do tratamento ${ }^{29}$.

Mediante isso, o apoio da família torna-se uma das principais medidas de suporte durante as fases de confirmação do diagnóstico e tratamento da patologia, assegurando-lhes maior autoestima e confiança no seu cuidado. É fundamental também, garantir o apoio psicoemocional por parte da equipe de saúde ${ }^{30-32}$.

\section{Expectativas sobre o futuro após diagnóstico de câncer de mama}

Para a maioria das
participantes do estudo, as
expectativas apontadas foram de
esperança e de que a cura iria
chegar, além de sentimentos de
gratidão e desejo de compartilhar
com outras mulheres a experiência
vivenciada, e ao mesmo tempo em
fortalecê-las para tais
enfrentamentos.

Pois é... aí eu fico pensando, meu Deus daqui pra frente como é que vai ser..., quanto tempo vou ficar assim, mas tenho fé que eu posso vencer, eu não vou dizer que eu vou morrer desse problema né filha, mais eu quero pedir a Deus que me dê mais tempo né, pra eu viver mais. (Andorinha, 73 anos)

Ah eu costumo falar assim para o meu marido, eu não faço planos, eu não tenho planos, eu falei pra ele, eu não quero fazer planos, eu quero viver um dia de cada vez, eu sempre falo pra ele vamos viver um dia de cada vez, viver, fazer o que a gente puder pelos meninos, pra mim é um dia de cada vez. (Calopsita 42, anos)

Não quero ficar assim como estou. Porque eles falaram que pode ter alguma sequela, alguma coisa né, então não quero ter sequela, quero ficar totalmente curada ... melhorar, não ter que tomar essa medicação de novo. (Beija Flor, 36 anos)

Minha filha a minha expectativa para o futuro, eu tenho pensado muito, não vou poder trabalhar mais, mas eu espero levar assim o que eu vivi de experiência para outras pessoas.... às vezes quando eu vou lá pegar remédio que eu estou o tomando... que eu tenho que tomar por 10 anos, eu vejo as pessoas todas sentadas naquelas cadeiras, fazendo a quimio né, então eu converso com todos, não desanima mulher... se alegra em Deus, porque se eu venci você vencerá também. (Bem Te Vi, 58 anos)

No que concerne as
expectativas de futuro das participantes do estudo, observa-se que elas demostraram ter expectativas positivas frente aos desafios e incertezas trazidos pela doença, fato esse que vai ao encontro com os achados de estudo realizado em Porto Alegre (RS), onde observou-se que mesmo com 
Sentimentos e expectativas de mulheres...

as intensas repercussões físicas e emocionais que o adoecimento e 0 tratamento desencadeou, elas desenvolveram perspectivas positivas em relação ao futuro, o que pode estar fortemente relacionado com a qualidade $\mathrm{da}$ informação que receberam no momento do diagnóstico por parte da equipe de saúde ${ }^{33}$.

Outro estudo realizado em sete hospitais para ao tratamento oncológico de mulheres com diagnóstico de câncer de mama nos Estados Unidos, com o intuito de avaliar os níveis de otimismo delas frente ao tratamento e perspectivas quanto ao futuro, identificou que a maioria delas também se mostrou otimista frente ao tratamento e com boas expectativas futuras ${ }^{34}$.

O tratamento do câncer de mama pode aumentar o risco de menopausa precoce e resultar em dificuldades reprodutivas. Isso tende a ser uma grande preocupação para muitos pacientes na idade reprodutiva frente ao futuro. Nesse sentido, estudo realizado na Universidade $\mathrm{da}$ Califórnia com 20 mulheres jovens, diagnosticadas com a doença, evidenciou que embora a fertilidade fosse importante para muitas participantes, as decisões de tratamento foram principalmente motivadas por preocupações com a sobrevivência e os anseios sobre a fertilidade dependiam das circunstâncias da vida de cada uma, como o fato de desejarem ou não serem mães ${ }^{35,36}$.

Em razão disso, é necessário melhorar as informações sobre o impacto do tratamento nas questões de fertilidade e sua preservação, mesmo que as preocupações não sejam expressas no momento do diagnóstico e início do tratamento oncológico. Pesquisas mostram que mulheres mais jovens parecem ter maiores necessidades psicossociais quando comparados a mais velhas, principalmente no que diz respeito a lidar com o impacto físico do tratamento e as consequências ginecológicas e reprodutivas associadas a ele $\mathrm{e}^{37,38}$.

Entretanto, após a vivencia do diagnóstico e tratamento da doença que para algumas é muito desgastante, é fundamental que profissionais de saúde possam incentivá-las e apoiá-las a voltar a 
sua rotina, principalmente o que se refere ao desenvolvimento de atividades sociais, profissionais e 0 cuidado consigo mesma39-41.

As limitações deste estudo estão relacionadas ao viés de memória, visto que algumas mulheres podem não ter respondido com exatidão sobre os sentimentos gerados frente ao diagnóstico da doença. Porém, essas limitações não invalidam os resultados encontrados e representam desafios a serem alcançados por pesquisas futuras.

\section{CONSIDERAÇÕES FINAIS}

Este estudo permitiu vislumbrar pontualmente os sentimentos e expectativas de mulheres frente ao diagnóstico de câncer de mama, dada a imensidão de emoções que podem ser manifestadas. Observou-se que o primeiro sintoma da doença foi a detecção de um nódulo e para algumas a confirmação do diagnóstico causou um grande impacto, suscitando sentimentos de negação, medo, incertezas e perspectiva de futuro incerto. 0 apoio familiar se configurou como umas das principais medidas de suporte ao longo do tratamento.

Ficou evidente a necessidade de apoio emocional não somente pela família, mas por parte dos profissionais desde a primeira busca por atendimento e ao longo do tratamento. Para tanto, recomenda-se que sejam efetivadas ações multiprofissionais de atenção a esse público, principalmente voltadas ao acolhimento humanizado e celeridade no processo de diagnóstico e início do processo terapêutico.

\section{REFERÊNCIAS}

1. Ministério da Saúde (BR). Instituto Nacional de Câncer José Alencar Gomes da Silva (INCA). O que é cancêr. Brasília (DF): Ministério da Saúde;2019.

2. Ministério da Saúde (BR). Síntese de resultados e comentários. Rio de Janeiro: Instituto Nacional de Câncer. Brasília (DF): Ministério da Saúde; 2016.

3. Ministério da Saúde (BR). Câncer de mama no Brasil: mortalidade está abaixo da 
média mundial. Brasília (DF): Ministério da Saúde; 2019.

4. Facina T. Diretrizes para a detecção precoce do câncer de mama no Brasil. Rev bras Cancerol. 2016; 62(1):59.

5. Vieira SC. Câncer de mama: Consenso da Sociedade Brasileira de Mastologia. Teresina: EDUFPI; 2016.

6. Carvalho CMS, Amorim FCM, Silva RTS, Alves VF, Oliveira ADS, Monte NS. Feelings of women diagnosed with breast cancer. Rev Enferm UFPE on line. 2016; 10(11):3942-50.

7. Cavalcante MLF, Chaves F, Ayala ALM. Câncer de mama: sentimentos e percepções das mulheres mastectomizadas. Rev bras ciênc saúde. 2016; 14(49):41-52.

8. Bardin L. Análise de conteúdo. São Paulo: Edições 70; 2011.

9. Magalhães $G$, Brandão-Souza $C$, Fustinoni SM, Matos JC, Schirmer J. Perfil clínico, sociodemográfico e epidemiológico da mulher com câncer de mama. J res fundam care Online. 2017; 9(2):473479.
10. Kudela E, Samec M, Kubatka P, Nachajova M , Laucekova Z, Liskova A, et al. Breast cancer in young women: Status Quo and advanced disease management through a predictive, preventive and personalized approach. Cancers. 2019; 11(11):1791.

11. Souza NHA, Falcão LMN, Nour GFA, Brito JO, Castro MM, Oliveira MS. Câncer de mama em mulheres jovens: estudo epidemiológico no Nordeste Brasileiro. Sanare. 2017; 16(2):60-67.

12. Siegel $R$, Jemal A. Cancer facts \& figures 2015. American Cancer Society Cancer Facts \& Figures; 2015.

13. Ministério da Saúde (BR). Instituto Nacional de Câncer José Alencar Gomes da Silva (INCA). Tipo de câncer. Brasília (DF): Ministério da Saúde; 2019. 14. Eugênio DSG, Souza JA, Chojniak R, Bitencourt AGV, Graziano L, Souza EF. Breast cancer features in women under the age of 40 years. Rev Assoc Med Bras. 2016; 62(8):755-761.

15. Torres-Reyes A, Tenahua-Quitl 
Sentimentos e expectativas de mulheres...

I, GarcíaLópez MA, PérezNoriega E, Cordero-Sánchez C, Ramos-Durán N, et al. Relación de calidad de vida $y$ autocuidado en pacientes adultos con cáncer. J Health NPEPS. 2019; 4(1):16-30.

16. Cirqueira TQP, Ferreira AGN, Santos MHS, Ferreira APM, Santos FDR, Pinheiro PNC. Relatos de vida de mulheres com câncer de mama. Atas Inv Cual en Salud. 2019; 2:1716-1724.

17. Ministério da Saúde (BR). Tipos de Cancêr: Mama. Brasília (DF): Ministério da Saúde; 2019.

18. Koo M, von Wagner C, Abel G, McPhail S, Rubin G, Lyratzopoulos G. Typical and atypical presenting symptoms of breast cancer and their associations with diagnostic intervals: evidence from a national audit of cancer diagnosis. Cancer Epidemiol. 2017; 48:140-146.

19. Redaniel M, Martin R, Ridd M, Wade J, Jeffreys M. Diagnostic Intervals and Its Association with Breast, Prostate, Lung and Colorectal Cancer Survival in England: Historical Cohort
Study Using the Clinical Practice Research Datalink. PLoS One. 2015; 10(5):e0126608.

20. Khakbazan Z, Taghipour A, Latifnejad Roudsari R, Mohammadi E. Help Seeking Behavior of Women with SelfDiscovered Breast Cancer Symptoms: A MetaEthnographic Synthesis of Patient Delay. PLoS One. 2014; 9(12):e110262.

21. Poum A, Promthet S, Duffy SW, Parkin DM. Factors Associated With Delayed Diagnosis of Breast Cancer in Northeast Thailand. J Epidemiol. 2014; 24(2):102-108.

22. Rosa LM, Radunz V. Significado do câncer de mama na percepção da mulher: do sintoma ao tratamento. Rev enferm UERJ. 2012; 20(4):445450.

23. Barros AES, Conde CR, Lemos TMR, Kunz JA, Ferreira MLSM. Sentimentos vivenciados por mulheres ao receberem o diagnóstico de câncer de mama. Rev enferm UFPE On. 2018; 12(1):102-11.

24. Mattias SR, Lima NM, Santos 
Sentimentos e expectativas de mulheres...

IDL, Pinto KRTF, Bernardy CCF, Sodré TM. Câncer de mama: sentimentos e percepções das mulheres diante do diagnóstico. $\mathrm{J}$ res fundam care online. 2018; 10(2):385-390.

25. Almeida TG, Comasetto I, Alves KMC, Santos AAP, Silva JMO, Trezza MCSF. Vivência da mulher jovem com câncer de mama e mastectomizada. Esc Anna Nery. 2015; 19(3):432438.

26. Ziguer MLPS, Bortoli CFC, Prates LA. Sentimentos e expectativas de mulheres após diagnóstico de câncer de mama. Espaç saúde. 2016; 17(1):108-113.

27. Costa RSL, Pessoa EP, Tavares EA, Queiroz GJC, Quinteiro J, Ribeiro MSA. Contexto psicossocial de mulheres mastectomizadas. Rev enferm contemporânea. 2019; 8(2):127-134.

28. Kunz JA, Conde CR, Lemos TMR, Barros AES, Ferreira MLSM. A religiosidade e espiritualidade de mulheres com câncer de mama submetidas a tratamento cirúrgico. Enferm atual. 2019;
86(24):1-9.

29. Tighe M, Molassiotis A, Morris J, Richardson J. Coping, meaning and symptom experience: A narrative approach to the overwhelming impacts of breast cancer in the first year following diagnosis. Eur J Oncol Nurs. 2011;15(3):226-32.

30. Conde CR, Lemos TMR, Pozati MPS, Ferreira MLSM. A repercussão do diagnóstico e tratamento do câncer de mama no contexto familiar. Rev UNINGÁ Rev. 2016; 47:95-100.

31. Ambrósio DCM, Santos MA. Apoio social à mulher mastectomizada: um estudo de revisão. Ciênc Saúde Coletiva. 2015; 20(3):851-864.

32. Teston EF, Fukumori EFC, Benedetti GMS, Spigolon DN, Costa MAR, Marcon SS. Sentimentos e dificuldades vivenciadas por pacientes oncológicos ao longo dos itinerários diagnóstico e terapêutico. Esc Anna Nery. 2018; 22(4):e20180017.

33. Ferreira VS, Salazar V, Peruchi RC, Donelli TMS, Castro EK. Vivências emocionais 
Sentimentos e expectativas de mulheres...

perspectivas de futuro em mulheres com câncer de mama. Psicol Hosp. 2015; 13(1):42-63.

34. Sonmezer M, Oktay K. Fertility preservation in young women undergoing breast cancer therapy. Oncologist. 2006;11(5):422-434.

35. Gorman JR, Usita P, Madlensky L, Pierce JP. Young Breast Cancer Survivors. Cancer Nursing. 2011; 34(1):32-40.

36. Thewes B, Butow P, Girgis A, Pendlebury $\mathrm{S}$. The psychosocial needs of breast cancer survivors; a qualitative study of the shared and unique needs of younger versus older survivors. Psycho-Oncology. 2004 ;13(3):177-189.

37. Wenzel L, Dogan-Ates A, Habbal R, Berkowitz R, Goldstein DP, Bernstein $M$, et al. Defining and measuring reproductive concerns of female cancer survivors. J Natl Cancer Inst Monogr. 2005; (34):94-8.

38. Matthews $\mathrm{EE}$, PF Cook. Relationships between optimism, well-being, selftranscendence, coping and social support in women during the treatment of breast cancer. Psychology. 2009; 18(7):716726.

39. Almeida TG, Comassetto I, Alves KMC, Santos AAP, Silva JMO, Trezza MCSF. Vivência da mulher jovem com câncer de mama e mastectomizada. Esc Anna Nery. 2015;19(3):432-438.

40. Santos IDL, Alvares RB, Lima NM, Matias SR, Cestari MEW, Pinto KR. Câncer de Mama: 0 apoio recebido no enfrentamento da doença. Rev enferm UFPE on line. 2017; 11(Supl. 8):3222-7.

41. Silva NR, Xavier HR, Rocha TL, Santos VLT, Mattos M, Santos DAS, et al. Perfil de saúde de mulheres atendidas em estratégias saúde da família em Mato Grosso. J Health NPEPS. 2019; 4(1):242-257. 
Conflito de interesses: Os autores declaram não haver conflito de interesses.

\section{Participação dos autores:}

- Concepção: Costa RSL, Lima RSM, Félix TC, Mota TMSC, Tavares EA, Queiroz GJC, Pereira EP.

- Desenvolvimento: Costa RSL, Lima RSM, Félix TC, Mota TMSC.

- Redação e revisão: Costa RSL, Lima RSM, Félix TC, Mota TMSC.

Como citar este artigo: Costa RSL, Lima RSM, Félix TC, Mota TMSC, Tavares EA, Queiroz GJC, et al. Sentimentos e expectativas de mulheres frente ao diagnóstico de câncer de mama. J Health NPEPS. 2020; 5(1):290-305.

Submissão: $27 / 10 / 2019$

Aceito: $11 / 03 / 2020$

Publicado: 01/06/2020 